\title{
Better Care in the Operating Room
}

\author{
Rajesh Aggarwal
}

Published online: 7 August 2014

(c) Société Internationale de Chirurgie 2014

The last 100 years have witnessed great advances in medicine with respect to antibiotic therapy, blood transfusion, organ transplantation, and more recently stem cell therapies and fetal surgery. Certainly this rate of progress has served society well with regard to life expectancy and quality of life. However, the past 25 years have noticed a new cause of illness - that of medical harm. Current estimates suggest that one in ten patients are harmed through their healthcare journey [1]. This has led to an emerging field of patient safety, whereby we can and must do better. Surgery has been in the limelight, as up to two-thirds of medical harm affects surgical patients [2].

In this issue of the journal, Kurmann et al. [3] describe a retrospective analysis of 117 patients undergoing elective open abdominal surgery at a single university-hospital center. At this hospital, senior and junior surgeons work together in 6-month blocks under a fellowship system. The intention was to study clinical outcomes for patients operated upon during month 1 versus month 6 for each pair of surgeons. Of the surgeries, 59 of them were conducted in month 1 of a 6-month block, and 58 during month 6 . Baseline characteristics of patients were similar between the two time points, as were operation duration and blood loss.

Notably, there were $36 \%$ fewer complications during month 6 than during month 1 , with an absolute reduction in complication rate of almost $20 \%$; the main decrease in complication rates was seen for those with Clavien-Dindo grades $<3$, i.e. not requiring re-intervention beyond additional medication, blood transfusion, or parenteral

R. Aggarwal $(\bowtie)$

Department of Surgery, McGill University Health Centre, 1650

Cedar Avenue, D6.136, Montreal, QC, Canada

e-mail: rajesh.aggarwal@mcgill.ca nutrition. Senior surgeons reported their concentration scores to reside above the overall mean from $37.5 \%$ of the time at month 1 , to almost $90 \%$ of the time during month 6 . Interestingly, they did not note an improvement in team collaboration scores between the two time periods.

Of note, this study is retrospective in nature, from a single center and included only five senior surgeons leading a total of 16 fellowship teams over a 2.5-year period. Thus, the results are preliminary at best, and warrant further investigation. The self-report data from senior surgeons and operative members was only collected for 26 of 117 procedures, and thus it is difficult to draw any meaningful conclusions. For the 26 selected procedures, the authors also measured sound levels above baseline, and report a significant reduction of $1.7 \mathrm{~dB}$ at month 6; whilst this is of statistical interest, the clinical impact is likely irrelevant.

Two similar studies were published in the surgical literature just last year, considering the impact of surgeon-tosurgeon familiarity in the operating room (OR) [4, 5]. The first considered coronary artery bypass graft (CABG) procedures, during which time on cardiopulmonary bypass (CBP) and cross-clamp time were measured [4]. CABG patients $(n=4,068)$ were operated upon by 11 attending surgeons and 73 cardiothoracic surgery fellows, over a 9 -year period. The mean number of cases between unique attending-fellow dyads was 10 (range 1-62). Whilst an additional year of attending experience only served to reduce CBP and cross-clamp times by about 20 seconds, each additional case performed by an attending-fellow dyad had a threefold effect in time reduction, up to the point where the 50th case performed by an attending-fellow dyad led to a $44 \%$ reduction in CBP time. A second study by the same group considered 754 bilateral reduction mammoplasty procedures, performed by eight attending 
and 107 assisting surgeons over a 12-year time period [5]. Compared with a new surgeon dyad, those with at least ten previous procedures together saved 16 minutes of OR time per case. However, this study noted no change in complication rate or patient satisfaction scores according to surgeon familiarity.

A criticism of all three studies is the focus upon the surgical micro-team or dyad, i.e. operating surgeon and assistant. Anesthesia and nursing colleagues are critical to clinical effectiveness and OR efficiency. Indeed, Kurmann et al. [3] noted that in month 6, neither the senior surgeons nor members of the entire OR team described any improvement to overall OR team collaboration scores. It could be said that, whilst the surgeon dyads worked better, this happened in isolation and there was no impact on the wider OR team.

Stepaniak et al. [6] considered patient outcomes, teamwork, and procedure duration for bariatric surgical operations performed at two general teaching hospitals, before and after the implementation of 'fixed OR teams', i.e. surgeons, residents, nurses, and anesthesiologist remained the same for the whole day. The hypothesis was that this produces a more stable atmosphere, better concentration, and quicker OR set-up and anticipatory instrument exchanges, resulting in improved surgeon confidence and better overall performance. Data from 1,387 patients (422 with variable OR teams, and 965 with fixed teams) led to a $30 \%$ reduction in complications (absolute reduction from 5.9 to $4.1 \%$ ), $27 \%$ increase in teamwork climate, $22 \%$ increase in safety climate, and $10 \%$ decrease in overall procedure time. Further gains of $5 \%$ in procedure time were achieved per repetition of the same procedure within the same day. This enabled OR planners to schedule more cases per day, without impacting upon quality of patient care.

It is suggested by these studies that OR team familiarity is related to efficient and high-quality care. So, how do we achieve similar results in our ORs? Advising patients to wait to schedule their operation until their surgeon dyad has worked together for 6 months, performed $50 \mathrm{CABG}$ procedures, or ten reduction mammoplasties together is ridiculous. The concept of fixed OR teams has merit, though it may lead to difficulty with regard to scheduling issues, especially in less specialized and lower-volume centers.

In 2006, the Veterans Health Administration in the USA implemented a national medical team training program across 130 hospital sites [7]. Team training included 2 months of preparation, a full-day on-site learning session with all OR staff (and closure of the OR for that day), and quarterly follow-up telephone calls to support, coach, and assess program implementation. A retrospective propensity-matched analysis of over 180,000 procedures revealed a $50 \%$ greater decline in the risk-adjusted surgical mortality rate in trained versus non-trained sites. This type of national program requires substantial investment, leadership, and a supportive environment. How then can we begin to facilitate such change for our patients in our own OR?

The World Health Organization (WHO) Safe Surgical Checklist is a good start, though its proper implementation and validity of outcomes has been brought into question recently [8]. In order to move ahead, we must begin to develop a robust, transparent, and inclusive system for working in the OR. If operative cases were agreed to be performed in a standardized manner, such as a series of steps with the same patient positioning, anesthesia and analgesia types, surgical instruments, etc., then it would not require an assisting surgeon 6 months, or 10-50 cases to 'learn' their attending's operating preferences. Surgeons, anesthesia, and nursing personnel could be prepared for every case, and understand all of the individual steps from beginning to end. Standards of care also lead to fewer lapses in the care pathway, such as from missed or erroneous steps. Whilst deviations will occur, they should be minimal and acknowledged appropriately.

For the OR to work as a system, it is critical that all OR staff, especially surgeons, emancipate the system and team approach. This will involve letting go of idiosyncrasies that abound when surgeons discuss their operative techniques, and, at least at an institution level, to agree upon operative processes that can be defined as the standard of care. Not only will this deliver better patient care, but will enable junior surgeons, anesthetists, and nurses to know what to expect in the OR, as well as to expedite and support their acquisition of skills. Standardized care processes in the OR will also enable equipment stocks to be monitored prospectively, and for procurement to occur more efficiently.

This goes beyond the OR setting, with critical aspects to pre-, intra- and post-operative processes being recently developed into enhanced recovery protocols [9]. Clinical staff, ward nurses, physiotherapy, nutrition, and social workers have become engaged to assist the patient through a standardized care pathway. Patients too are actively encouraged to participate in their care, such as how much to drink, how often to walk, and when to do their breathing exercises. The ability to bring the team together is only possible because of the approach is standardized, though deviations from care can still occur. Teams of clinicians, nursing staff, and allied healthcare workers have come together to define and agree upon standard pathways of care, and when implemented, has been shown to lead to reduced complication rates, shorter lengths of stay, and economic benefits.

As surgeons, it is important to understand that we are working in a part of a system of care that aims to provide 
high-quality treatment for our patients. The investigation by Kurmann et al. [3] seeks to develop a closed system of care every 6 months; the imperative should be to define the provision of best care for every patient, at every time, regardless of surgeon or OR team familiarity. This type of system is exactly what airline crews, construction site workers, culinary and military teams ascribe to, whereby roles are pre-specified, competencies known, and mission objectives defined [10]. Whilst surgery is certainly complex, evidence-based and standardized operative care plans are possible, with the potential for translation from the institution level to regional and national imperatives. The ultimate gain shall be to deliver safer care with translation to superior outcomes for our patients.

Conflict of interest Rajesh Aggarwal receives consulting fees from Applied Medical.

\section{References}

1. de Vries EN, Ramrattan MA, Smorenburg SM, Gouma DJ, Boermeester MA (2008) The incidence and nature of in-hospital adverse events: a systematic review. Qual Saf Health Care 17(3):216-223
2. Gawande AA, Thomas EJ, Zinner MJ, Brennan TA (1999) The incidence and nature of surgical adverse events in Colorado and Utah in 1992. Surgery 126(1):66-75

3. Kurmann A, Keller S, Tschan-Semmer F, Seelandt J, Semmer NK, Candinas D, Beldi G (2014) Impact of team familiarity in the operating room on surgical complications. World J Surg. Epub 3 Jul 2014

4. Elbardissi AW, Duclos A, Rawn JD, Orgill DP, Carty MJ (2013) Cumulative team experience matters more than individual surgeon experience in cardiac surgery. J Thorac Cardiovasc Surg 145(2):328-333

5. Xu R, Carty MJ, Orgill DP, Lipsitz SR, Duclos A (2013) The teaming curve: a longitudinal study of the influence of surgical team familiarity on operative time. Ann Surg 258(6):953-957

6. Stepaniak PS, Heij C, Buise MP, Mannaerts GH, Smulders JF, Nienhuijs SW (2012) Bariatric surgery with operating room teams that stayed fixed during the day: a multicenter study analyzing the effects on patient outcomes, teamwork and safety climate, and procedure duration. Anesth Analg 115(6):1384-1392

7. Neily J, Mills PD, Young-Xu Y, Carney BT, West P, Berger DH, Mazzia LM, Paull DE, Bagian JP (2010) Association between implementation of a medical team training program and surgical mortality. JAMA 304(15):1693-1700

8. Leape LL (2014) The checklist conundrum. N Engl J Med 370(11):1063-1064

9. Kehlet H, Wilmore DW (2008) Evidence-based surgical care and the evolution of fast-track surgery. Ann Surg 248(2):189-198

10. Gawande AA (2012) Big med. The New Yorker. 6 Aug 2012 